\title{
MODELOS DE RESPONSABILIDAD SOCIAL UNIVERSITARIA Y PRINCIPALES DESAFÍOS PARA SU IMPLEMENTACIÓN EN FACULTADES DE NEGOCIOS
}

\author{
UNIVERSITY SOCIAL RESPONSIBILITY \\ MODELS AND THE MAIN CHALLENGES FOR \\ IMPLEMENTATION IN BUSINESS SCHOOLS
}

\author{
Javiera N. Müller Núñez ${ }^{1}$, Freddy Coronado Martínez²
}

\section{RESUMEN}

La responsabilidad social (RS) se ha convertido en un fenómeno global por los múltiples eventos que transgreden los límites de una aceptable convivencia social y respetuosa con el desarrollo sostenible, asociados a desempeños poco éticos. Por esto, es transcendental que la RS esté arraigada en las creencias de los futuros líderes de las empresas y sociedad. Las universidades no pueden abstraerse de esta necesidad y menos ante un contexto que nos muestra, una y otra vez, que sus egresados tienen que asumir el liderazgo y generar cambios necesarios para que la sostenibilidad no sea solo anhelo, sino también una realidad.

El objetivo del presente trabajo es estudiar los modelos de Responsabilidad Social Universitaria (RSU) y presentar los desafíos para su implementación principalmente en Facultades de Negocios. Para cumplir este objetivo, se darán a conocer los principales modelos existentes de RSU y se relacionarán entre sí, para determinar similitudes o diferencias.

Al analizar los modelos y contrastándolo con la realidad Chilena, se presentan los desafíos para la implementación de la RSU en instituciones de educación superior. Finalmente, se muestran algunos ejemplos de iniciativas especialmente diseñadas para Facultades de Negocios.

Entre los resultados, es posible destacar el reconocimiento de la necesidad de que la RSU sea una prioridad a nivel estratégico, y desde ahí incorporar una dirección de RSU que incluya recursos para dar curso a este tipo de iniciativas. También se destaca la necesidad de incorporar reportes de RSU periódicos a cargo de centros de responsabilidad específicos para su transparencia, comparabilidad, seguimiento y cumplimiento.

Palabras claves: Facultades de Negocios, Modelos, Responsabilidad Social Universitaria, Sostenibilidad Universitaria.

Recepción: 10/11/2016. Aprobación: 29/12/2017.

\section{ABSTRACT}

Social responsibility (SR) has become a global phenomenon due to multiple events that transgress the boundaries of acceptable social and respectful coexistence with sustainable development, associ-

1 Facultad de Economía y Negocios, Universidad Santo Tomás, Santiago, Chile.jmullern@santotomas.cl.

2 Facultad de Economía y Negocios, Universidad de Chile, Santiago, Chile. fcoronadom@fen.uchile.cl 
ated with unethical performance. Therefore, it is important that the (SR) is rooted in the beliefs of the future leaders of business and society. Universities can not be abstracted from this need in a context that shows us again and again, that their graduates have to take the lead and generate changes needed so sustainability will not only be a wish, but also a reality.

The objective of this work is to study models of University Social Responsibility (USR) and to present the challenges for implementation, mainly in business schools. To meet this goal, we both introduce the main existing models of USR and relate to each other, to determine similarities or differences.

By analyzing the models and contrasting them with the Chilean reality, we present the challenges for the implementation of the USR in higher education institutions. Finally, some examples of initiatives specifically designed for business schools are presented.

Among the results, we can highlight the recognition of the need for the USR is a priority at the strategic level, and as a result, the need to introduce an office of USR that has resources to implement such initiatives. There is also a need for introducing USR periodic reports by responsibility centers for ensuring transparency, comparability, monitoring and compliance.

Keywords: Business Schools, Models, University Social Responsibility, University Sustainability.

\section{INTRODUCCIÓN}

Desde la Primera Cumbre de la Tierra (1972) se marcó un punto de inflexión en el desarrollo de la política internacional del medio ambiente y se comenzó a dialogar sobre la responsabilidad social. Luego, en la Conferencias de la ONU sobre el Medio Ambiente y el Desarrollo (1992) se tomó en consideración a nivel internacional el concepto de desarrollo sostenible en el tiempo, es decir, respecto al desarrollo capaz de satisfacer las necesidades actuales sin comprometer los recursos para las próximas generaciones.

Asimismo, en la renombrada declaración de Talloires (1990), que fue firmada por rectores de más de cuatrocientas universidades del mundo, junto con la Declaración Mundial sobre la Educación Superior en el Siglo XXI de la UNESCO (1998), exponen a la década 2005-2014 como la de educación para el Desarrollo Sustentable. Asimismo, en septiembre de 2015 se llevó a cabo la cumbre del desarrollo sostenible, en el cual se vuelve a destacar el rol de la universidad para fomentar la sostenibilidad.

Desde el punto de vista de la Responsabilidad Social Universitaria, las universidades deben contar con un diseño curricular que incorpore y considere los desafíos de la sostenibilidad. En segundo término, se deben implementar métodos de enseñanza y aprendizaje que fomenten el desarrollo de actuaciones profesionales responsables. Como tercer elemento, es fundamental el desarrollo de investigaciones e innovaciones en esta materia. Finalmente, las universidades debieran contar con un modelo de gestión universitario que incorpore estos lineamientos de tal forma, que la propia universidad se preocupe responsablemente de sus impactos.

Es importante destacar que en particular las Facultades de Negocios tienen especiales desafíos por formar personas, principalmente, porque egresan de este tipo de facultades individuos que se vincularán y tomarán decisiones administrando organizaciones (Kaivola \& Rohweder, 2007). Mediante esto se podrá disminuir las probabilidades de que las futuras generaciones tomen decisiones que puedan causar externalidades negativas al resto de la sociedad, además disminuir posibilidades de colusión, mala utilización de recursos y escándalos financieros, entre otros. Por lo tanto, como objetivo de este ensayo es conocer los modelos de Responsabilidad Social Universitaria existentes, relacionarlos entre sí para encontrar similitudes o diferencias y también investigar los desafíos para su implementación en las universidades, en especial a Facultades de Negocios.

\section{DESARROLLO}

El gran exponente sobre la Responsabilidad Social Universitaria (RSU), Vallaeys (2006) la define como "Una política de calidad ética del desempeño de la comunidad universitaria (estudiantes, docentes y personal administrativo) a través de la gestión responsable de los impactos educativos, cognitivos, laborales, sociales y ambientales que la universidad genera, en un diálogo participativo con la sociedad para promover el desarrollo humano sostenible", es decir, gestionar responsablemente todos los impactos 
de la universidad siempre con la participación activa de la sociedad.

Para conocer las aristas de la implementación de RSU, es importante estudiar los modelos existentes relacionados con ésta, estos permiten la gestión de iniciativas en las áreas correctas y conocer los posibles impactos que pueden generar, además entregan los lineamientos para conocer los pasos a seguir para su implementación, guían el monitoreo de ésta, y finalmente se identifican las mejores prácticas que faciliten el aprendizaje continuo.

En este ensayo, se revisarán los principales modelos existentes y renombrados relacionados a la RSU, entre ellos el modelo de Vallaeys
(2006), Universidad Construye País (2006) y AUSJAL (2009), luego se analizará la relación entre los modelos y los desafíos que presentan para su implementación.

\section{Modelo Vallaeys (2006, 2009, 2013)}

El modelo de Vallaeys para la RSU sigue la lógica de las áreas contenidas en las universidades, además incluye los actores interesados en cada área y los posibles impactos que puede generar. Los impactos de la universidad pueden ser agrupados en impactos organizacionales, educativos, cognitivos y sociales (Vallaeys, 2006), los cuales se muestran en la figura 1.

Figura 1. RSU modelo Vallaeys.

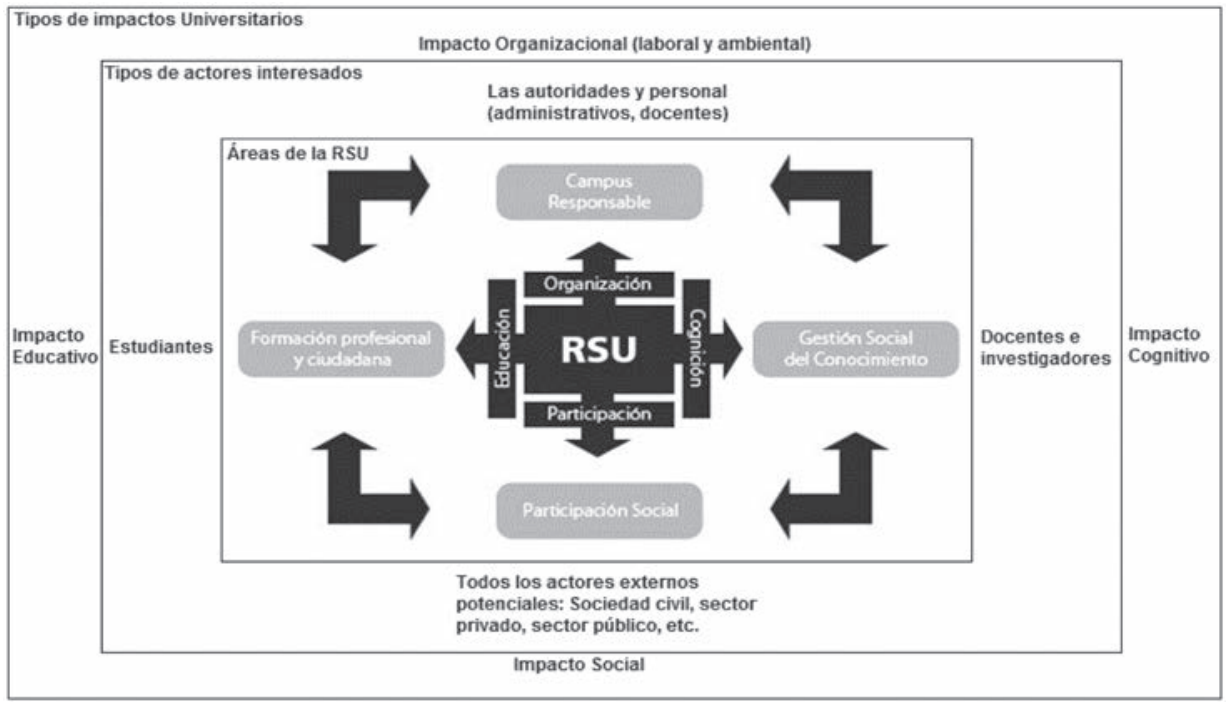

Fuente: Creación propia a partir de Vallaeys (2006, 2009 y 2013).

En este modelo existen cuatro áreas que vinculan a ciertos stakeholders y se explicará el posible impacto sobre ellos. El impacto educativo implica la gestión socialmente responsable de la formación académica, es decir, que la orientación curricular tenga una relación estrecha con los problemas reales de la sociedad. Se espera que los alumnos egresen con valores éticos, habilidades blandas y sociales como la competencia de responsabilidad social arraigada dentro de sus creencias, además de las competencias profesionales necesarias. En este impacto, los principales actores interesados son los estudiantes, ya que son los que se benefician al mejorar este impacto, y el área de RSU es la denominada formación profesional y ciudadana.

El impacto organizacional, que implica promover un comportamiento organizacional responsable con todos los integrantes de la comunidad universitaria, se divide en dos: el impacto laboral que afecta la vida del personal (administrativo y docente), y el impacto ambiental (desechos, desforestación, transporte, etc.). En este impacto los principales actores involucrados son el personal, como docentes y administrativos, ya que son los que se benefician al mejorar este impacto, y el área de RSU denominada es la de campus responsable.

Luego, el impacto de cognición es aquel provocado en la producción del conocimiento; ésta, 
según Vallaeys (2006), es la categoría más importante, ya que a través de dichos conocimientos y paradigmas los futuros líderes construyen a la sociedad, para esto debe existir una gestión socialmente responsable de la producción y difusión del saber, es decir la investigación y los modelos epistemológicos promovidos desde las escuelas y en el aula. La universidad debe desarrollar conocimientos entre sus estudiantes, que estén acorde a las necesidades de la sociedad y el país. En este impacto los principales actores involucrados son los docentes e investigadores, ya que son los que se encargan de mejorar este impacto, y el área de RSU denominada es la de gestión social del conocimiento.

Finalmente, se encuentra el impacto social que es aquel generado para el desarrollo de la sociedad de una forma bidireccional, es decir la entrega de conocimiento y ayuda desde la uni- versidad a la sociedad y entrega de conocimiento desde la sociedad a la universidad, por ejemplo la realización de proyectos con otros actores del tal modo que se constituyan vínculos para el aprendizaje mutuo y el desarrollo social. En este impacto los principales actores involucrados son los agentes externos potenciales, como otras universidades, comunidad cercana como vecinos, comunidad científica, otras universidades, familiares del personal y estudiantes, etc., ya que son los protagonistas al realizar iniciativas en este impacto, y el área de RSU se denomina participación social.

Adicionalmente, el modelo de los primeros pasos de Vallaeys (2009) explica cómo se debería implementar la RSU en las universidades, para esto incluye cuatro pasos detallados en la figura siguiente:

Figura 2. Sistema de cuatro pasos para la RSU.

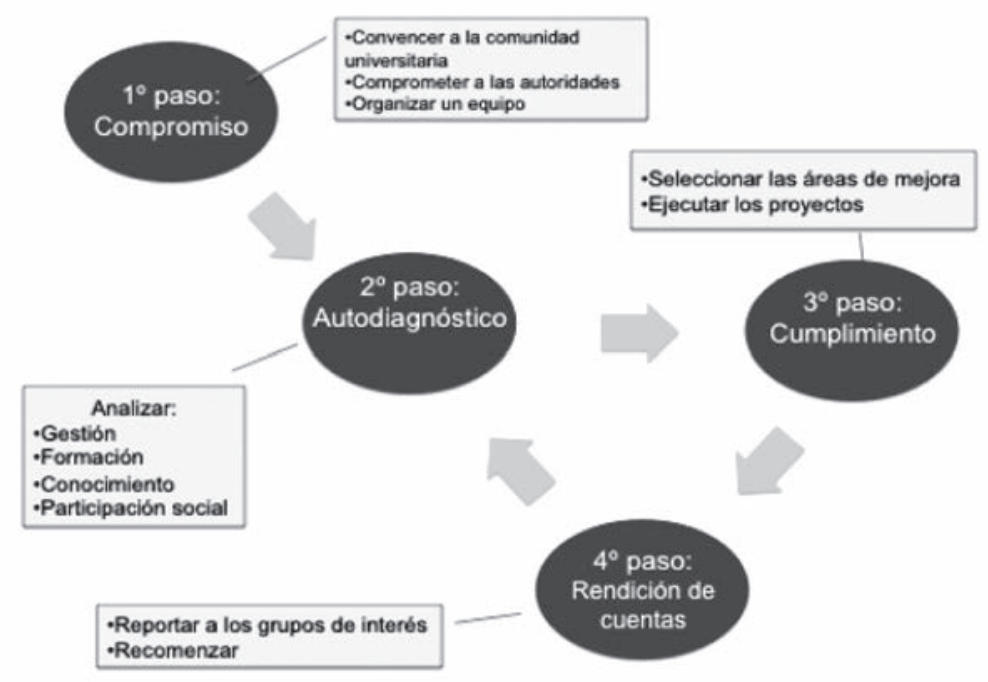

Fuente: Vallaeys (2009).

El primer paso es el del compromiso que se refiere a la articulación de la RSU con el proyecto institucional, y las declaraciones estratégicas de la universidad. Exige un claro compromiso de la alta dirección y la implicación de toda la comunidad universitaria (docentes, estudiantes, administrativos y autoridades), así como la creación de un equipo o dirección a cargo del tema para el diagnóstico institucional y encargado de conseguir los recursos para fomentar y gestionar las iniciativas de RSU en la institución.
Luego, el segundo paso del autodiagnóstico son las herramientas cuantitativas y cualitativas para el diagnóstico de los cuatro ámbitos clave de las universidades (ámbito de la gestión de la organización, ámbito de la formación educativa, ámbito del conocimiento y la investigación, y ámbito de la participación social). Con esto se conocerá la realidad de la universidad en temas de RSU.

El tercer paso, del cumplimiento, tiene como objetivo contrastar los resultados del diagnós- 
tico con las declaraciones de la universidad, planificar las áreas de mejora y ejecutar los proyectos de responsabilidad social, siempre con la más amplia participación de los miembros de la comunidad universitaria y los actores externos pertinentes.

Finalmente el cuarto paso, de rendición de cuentas, ofrece algunas ideas para evaluar y comunicar de forma transparente los resultados de los proyectos de mejora institucional, afinar las estrategias y reiniciar el ciclo concentrándose en los aspectos que hayan presentado las mayores dificultades.

Se puede argumentar que para que una organización se considere socialmente responsable debe cumplir con estos cuatro pasos, es decir, debe llegar a informar sus acciones con veracidad siendo transparente y consistente en la rendición de cuentas o accountability.

"Lo que no se mide no se puede controlar" es una frase de William Pepperell Montague, filósofo estadounidense que hace mucho sentido en este estudio ya que es muy importante poder medir la implementación de la RSU en todos sus impactos, para verificar su avance y retroalimen- tación. Para esto, el modelo de Vallaeys (2009) también define indicadores de auto diagnóstico cuantitativos, en relación a la gestión organizacional.

En resumen Vallaeys (2009) define un modelo de RSU robusto con una guía de primeros pasos para implementación de la RSU y también indicadores genéricos para medir los impactos de sus cuatro dimensiones.

\section{Modelo Universidad Construye País (2006)}

El proyecto Universidad Construye País (UCP) parte el 2001 desde el diagnóstico lamentable que indica que las universidades chilenas no tenían en el centro de sus misiones temáticas tales como la pobreza, la desintegración social, el capital social o el desarrollo sustentable.

El principal objetivo del proyecto UCP es expandir el concepto y la práctica de la responsabilidad social dentro del sistema universitario chileno. Para esto se unieron 13 universidades chilenas ${ }^{3}$ :

A continuación, en la figura 3 se mostrará cómo se conforma el modelo de la UCP.

Figura 3. Modelo RSU para Universidad Construye País.

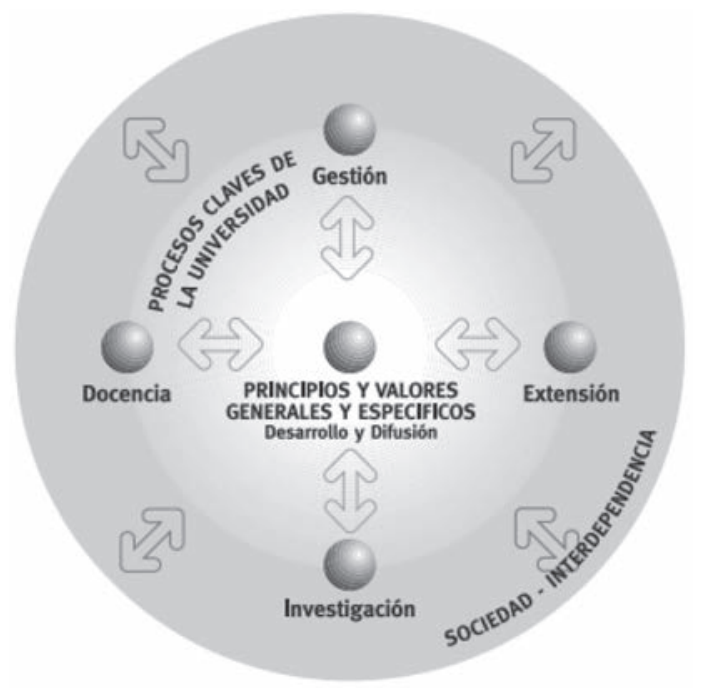

Fuente: Universidad Construye-País (2006).

La universidad se efectúa a través de tres funciones tradicionales, que son docencia, investigación y extensión, y además de una actividad indispensable en toda organización que es su gestión. En el centro de estos cuatro procesos claves se encuentran los principios y valores, como el sol del sistema, es decir esos cuatro procesos del quehacer universitario deben estar ilu- 
minados por principios y valores (Universidad Construye-País, 2006). Existen valores del plano personal como: Dignidad de la persona, integridad y libertad. Valores del plano social como: Bien común, equidad social, desarrollo sostenible, cuidado del medio ambiente y solidaridad para la convivencia, aceptación y aprecio a la diversidad y ciudadanía, democracia y participación. Finalmente valores del plano universitario como: Compromiso con la verdad, excelencia, interdependencia e interdisciplinariedad. Por lo tanto, el valor agregado de este modelo son las definiciones de valores y principios como centrales en la RSU.

\section{Modelo de AUSJAL (2009)}

La Asociación de Universidades confiadas a la compañía de Jesús en América Latina propone un cuadro para la autoevaluación de la RSU en las universidades (AUSJAL, 2009), este se presenta a continuación en la figura 4 .

Figura 4. Modelo de RSU por AUSJAL.
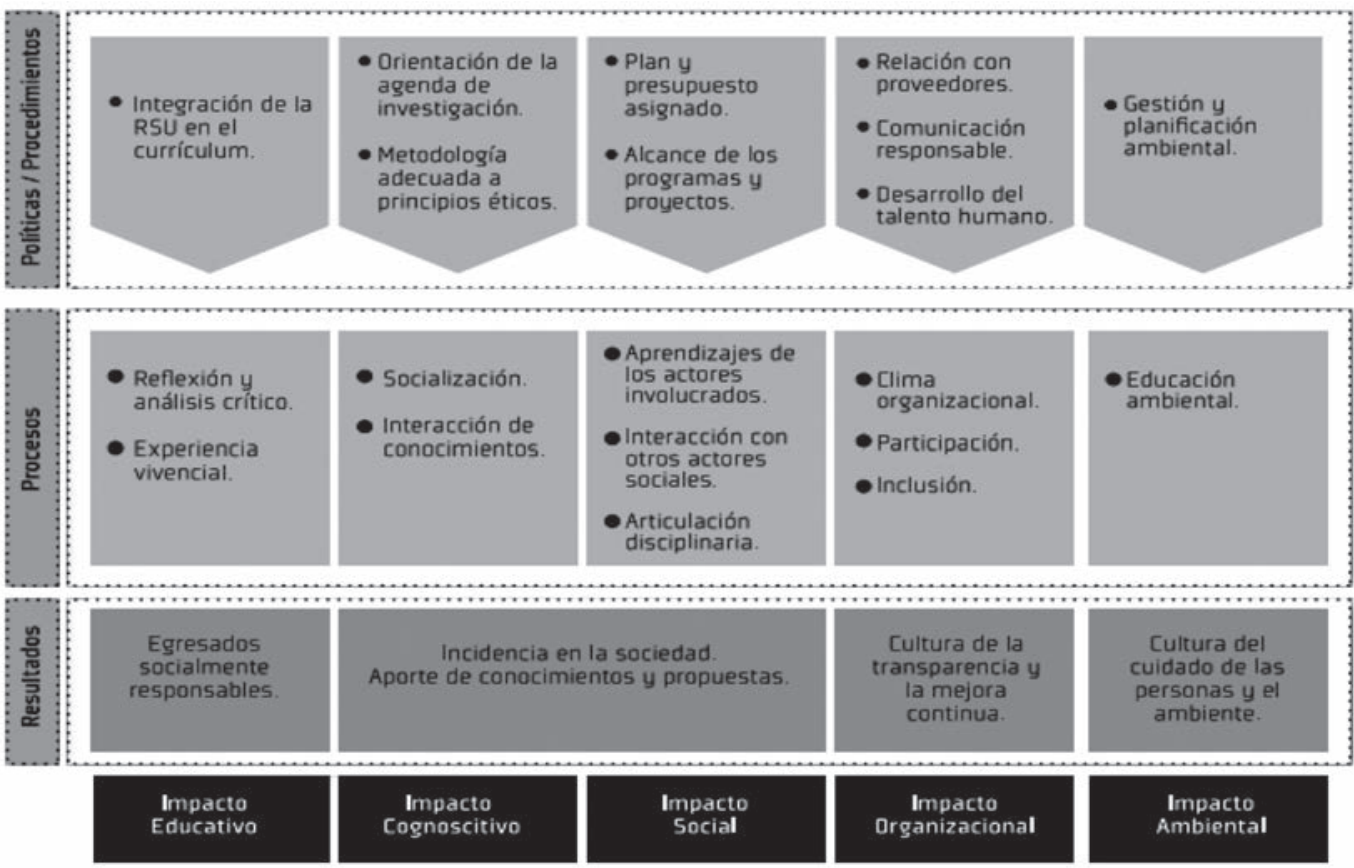

Fuente: AUSJAL (2009).

Este modelo tiene cinco áreas de impacto que se explican a continuación:

Impacto educativo: La universidad tiene un impacto sobre la formación de los estudiantes, su manera de entender e interpretar el mundo y su relación con la forma como se comportan y valoran ciertos sucesos en su vida. Por ello, la universidad debe procurar desde este enfoque, la gestión socialmente responsable de la formación académica y la pedagogía, propiciando experiencias vivenciales, iniciativas interdisciplinarias y reflexión crítica de las mismas.

Impacto cognoscitivo: La universidad orienta la producción del saber, por ello debe procurar desde este enfoque la gestión socialmente responsable de dicha, a fin de evitar la fragmen- tación del saber, favorecer la articulación entre tecnología, ciencia y sociedad, e influir fuertemente en la definición y selección de los problemas de la agenda científica.

Impacto social: La universidad impacta sobre la sociedad y su desarrollo económico, social y político, no sólo porque forma profesionales y líderes, sino porque ella misma es un referente y actor social. Por ello, la universidad debe procurar la gestión socialmente responsable de su participación en el desarrollo humano sustentable de la comunidad de la que forma parte.

Impactos de funcionamiento organizacional: Como cualquier organización, la universidad genera impactos en la vida de los miembros de la comunidad universitaria. Por lo tanto, desde 
este enfoque se debe procurar la gestión socialmente responsable de la organización misma de manera coherente con los principios institucionales en un ambiente que favorezca la inclusión, la participación y la mejora continua.

Impactos ambientales: La universidad como el resto de las organizaciones genera impactos sobre el medio ambiente que afectan su sustentabilidad a nivel global. Por ello, la universidad debe contribuir a crear una cultura de protección del ambiente y procurar la gestión socialmente responsable de los recursos ambientales dispo- nibles para las generaciones actuales y futuras.

Finalmente este modelo aporta como valor agregado la inclusión de una lógica de inputproceso-output el ordenamiento de sus objetivos para el cumplimiento.

\section{Análisis de los modelos de RSU}

Al relacionar los modelos de RSU se puede apreciar que siguen una misma lógica de los cuatro impactos universitarios, en la siguiente figura se relacionan los tres modelos estudiados:

Figura 5. Relación modelos de RSU.

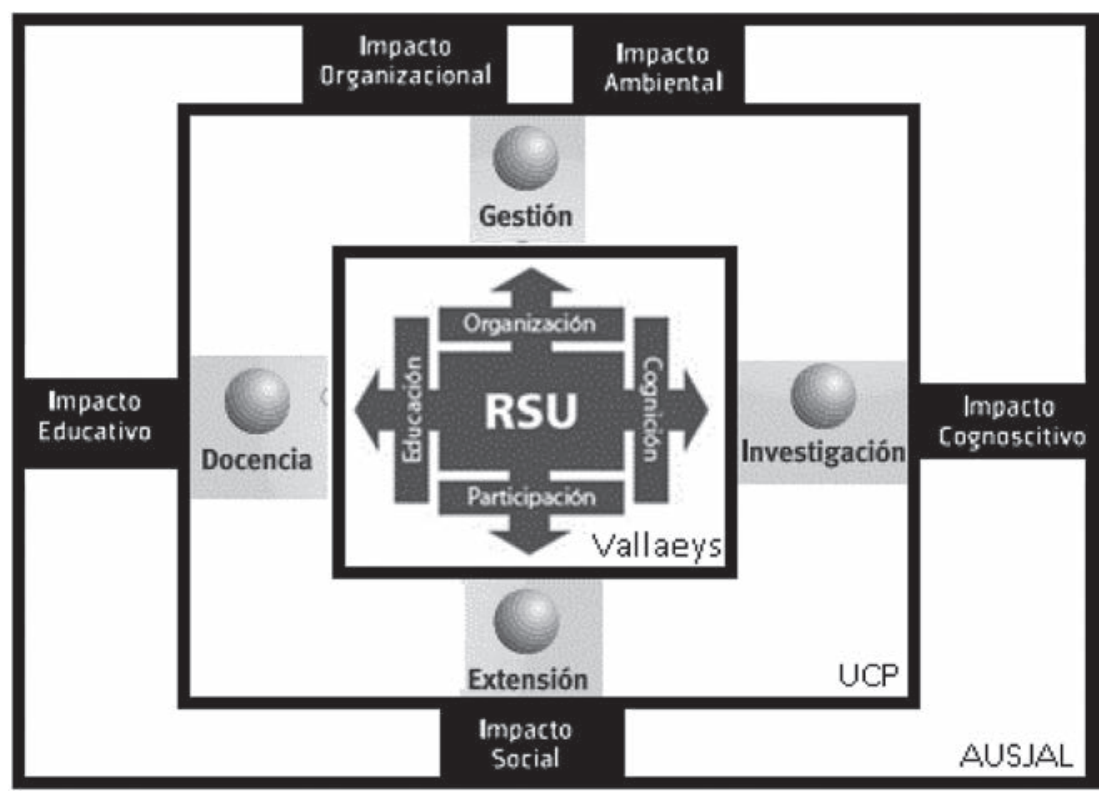

Fuente: Creación propia.

Para el modelo de Vallaeys (2006) el área de la educación es similar a lo que explica UCP (2006) con la dimensión de docencia y el modelo de AUSJAL (2009) con el impacto educativo. Todos tienen relación con la formación académica, es decir, que la orientación curricular tenga relación con los problemas de la sociedad.

Luego, el modelo de Vallaeys (2006) tiene una dimensión de impacto en la cognición que implica la gestión responsable del conocimiento, lo que hace referencia a la difusión del saber, la investigación científica y paradigmas que constituye la sociedad, asemejándose al impacto cognoscitivo del modelo AUSJAL (2009) y a la parte de investigación de UCP (2006).

Siguiendo con la semejanza, para Vallaeys
(2006) la participación social implica tener proyectos con otros actores sociales que es exactamente lo mismo que el impacto social de AUSJAL (2009) y muy parecido a lo que se refiere con extensión de UCP (2006).

Finalmente, se encuentra el área de la organización, que se traduce en el impacto responsable sobre el medio ambiente, la comunidad y la vida del personal, y también en que las acciones del personal sean responsables de forma consistente a la RSU, que es lo mismo que el impacto ambiental de AUSJAL (2009) y la dimensión de gestión del UCP (2006).

Así, luego de estudiar los modelos existentes de RSU se puede notar que siguen un mismo eje, además el modelo de UCP (2006) y AUSJAL 
(2009), dado su bibliografía, se basan en el de Vallaeys (2006).

Entre las diferencias están que el modelo de Vallaeys (2006) incluye pasos para la implementación, luego el modelo de UCP (2006) incluye la importancia de los valores como sol del sistema y finalmente el modelo de AUSJAL (2009) incluye la lógica de input proceso y output para el cumplimiento de la RSU.

Es importante mencionar que el modelo de Vallaeys muestra también ideas genéricas de pasos para implementar RSU, por lo que no existen diferencias culturales significativas en su modelo con la realidad chilena que concluya una barrera para su utilización en universidades incipientes. Por lo tanto, se concluye que el modelo de Vallaeys (2006) es el mejor representante de los modelos de RSU, ya que es el más antiguo e influyente en la literatura de RSU, además reconocido y robusto ya que también considera un sistema de cuatro pasos para la implementación de RSU.

\section{Desafíos para su implementación}

Para fomentar el desarrollo de la RSU se deben fomentar las 4 áreas que impactan según los modelos estudiados, en la realidad universitaria la mayoría de estas iniciativas nace por parte de pequeños grupos de alumnos para responder a las necesidades sociales y ambientales, y algunos profesores que incorporan por ejemplo, en sus cursos algunas temáticas de la RS.

Sin importar el modelo estudiado, todos los modelos coinciden en que la implementación de la RSU requiere esfuerzos integrales en las múltiples áreas de la gestión universitaria: docencia, investigación, gestión y participación social. Por lo tanto, se requiere el compromiso del equipo directivo para la apropiada implementación y dejar con un rol estratégico a la RSU. Esfuerzos aislados, sostenidos por actores voluntarios pero que no tienen la capacidad de aportar transversalmente (ejemplo: afectar el proceso formativo de todos los estudiantes de la facultad en cuestión), no representan esfuerzos institucionales por hacer de la RS parte de la formación.

Una vez asumido el compromisos del equipo directivo, esto debe ser acompañado de recursos para facilitar la implementación de las nuevas prácticas. Por ejemplo, si la intención es fortalecer la investigación, entonces se requerirán incentivos como fondos concursales o premios que fomenten la investigación en RS. Por otro lado, si la intención es fortalecer la docencia en RS, el apoyo a cursos de manera transversal debiese ser implementado para asegurar que todos los estudiantes que transitan por el proceso formativo, tienen dichas intervenciones como parte del currículo. Por ejemplo, si hay un curso en particular en el cual se implementa metodología de aprendizaje y servicio, esto debiese ocurrir en todas las secciones de dicho curso. Esto podría requerir por ejemplo capacitación de todos los profesores y ayudantes, además de un manejo de manera centralizada (escuelas de pregrado) de la relación con los socios comunitarios respectivos (ej.: PYMES involucradas en el proceso), además de realizar esfuerzos por mantener actualizada la base de datos de actores interesados.

El aprendizaje que requiere la implementación de la RSU implica involucrar a todos los actores en procesos colectivos de reflexión. Sin embargo, este proceso aunque necesario, se ve entrampado por la estructura universitaria que muchas veces deposita los esfuerzos en unidades que no tienen canales de comunicación formales. Por ejemplo, es recurrente la falta de comunicación entre las escuelas de pregrado encargadas del proceso formativo y las unidades de extensión encargadas de la relación con la sociedad. Por otra parte, es común ver también, una separación entre aquellos profesores que dedican mayor tiempo a la docencia, en quienes suele recaer la demanda formativa con este tipo de innovaciones, y aquellos académicos que dedican mayor parte del tiempo a la investigación.

Asimismo, se da cuenta de un desafío importante como realizar rendición de cuentas, muy pocas universidades utilizan reportes de sostenibilidad o de RSU, y las que lo utilizan no siempre tienen una periodicidad de reportes estables, por lo que no permite su comparación a lo largo del tiempo entre la misma universidad ni tampoco entre distintas universidades. Es importante mencionar que no existe un formato apropiado de reportabilidad para la RSU, ya que el formato comúnmente utilizado por las empresas GRI (Global Reporting Initiative) no considera áreas de docencia e investigación como necesarias para la rendición de cuentas de una universidad.

Algunas iniciativas que se pudiesen promover como desafíos primordialmente para las Facultades de Negocios son las siguientes.

Impacto educativo: Se pueden fomentar cursos obligatorios y electivos con metodologías pedagógicas como el Aprendizaje-Servicio y Aprendizaje basado en los Desafíos Profesiona- 
les y Empresariales relacionados a la responsabilidad social; esto podrían ser cursos que tengan iniciativas como asesorías para las empresas u organizaciones participantes. Este tipo de metodologías podrían ir incluidas en cursos afines como Fundamentos de Costos, Marketing, Evaluación Proyectos, Recursos Humanos, Seminario de integración profesional, entre otros. Estas metodologías permiten exponer al alumno a un proceso de enseñanza-aprendizaje que mezcla la teoría y la práctica en una problemática social y profesional significativa.

Además, es recomendable el perfeccionamiento permanente de académicos con respecto a temas de responsabilidad social y sostenibilidad para que pudiese ser transmitido a los alumnos.

Impacto social: Se pueden mencionar proyectos y/o asesorías estudiantiles con colegios, municipalidades y microempresarios dónde el diseño y la ejecución de la actividad debe ser consistente con criterios de RS. Para que esto sea sostenible en el tiempo, se recomienda que se gestione una base de datos constantemente actualizada de socios estratégicos que permitan dar continuidad a estos proyectos, como pequeños empresarios, comunidades y/o empresas.

Impacto cognoscitivo: Fomentar tesis vinculadas a la Responsabilidad Social y de investigación aplicada a problemáticas sociales. Para esto se sugiere implementar incentivos para el fortalecimiento de la investigación.

Impacto organizacional: Fomentar buenas prácticas de gobierno universitario, mejorar la comunicación entre los actores involucrados, realizar iniciativas para fomentar el desarrollo del capital humano, mejorar inclusión, además se puede implementar un sistema de gestión, difusión y educación para un campus sustentable, como por ejemplo fomentar el reciclaje, tratamiento de residuos, de utilización de recursos como luz, papel y agua, entre otros.

\section{CONCLUSIÓN}

Para que la RSU sea un tema de importancia dentro de la universidad, como primer paso del sistema de Vallaeys (2009), esta debe ser parte del compromiso de la organización, es decir, debe estar incluida como un rol estratégico dentro de su plan estratégico o declaraciones. Con esto, se podrán construir los lineamientos para desarrollar iniciativas y herramientas para su diagnóstico y mejora continua. Vallaeys (2009) en su modelo explica en este paso que se debe oficializar el compromiso institucional con RSU, comunicando la RSU para lograr una participación, designando un equipo encargado de RSU para el diagnóstico y coordinación.

Los esfuerzos en RSU deben ser integrales y consistentes en todas las áreas de RSU, es decir no se pueden tener asignaturas de responsabilidad social, si la universidad no se gestiona responsablemente, debe existir una coherencia para inculcar realmente la RS.

También se muestra que las universidades deben realizar mediciones de sus iniciativas, para conocer lo que están haciendo y poder gestionarlo, incluyendo recursos para las iniciativas, por lo que deben tener indicadores, metas y resultados. Con esto conocerán el impacto y podrán rendir cuentas a la sociedad, transparentando la información a las partes interesadas. Asimismo, es importante que la rendición de cuentas sea transparente, constante y sostenible en el tiempo para así poder conocer si existen mejoras en el tiempo, además de favorecer la comparabilidad entre la misma institución y otras instituciones para los stakeholders.

En general, todas las universidades realizan acciones socialmente responsables, por lo que en primera instancia cuando se diagnostiquen se espera que tengan por lo menos algunas iniciativas en todas las dimensiones. También se espera que esa medición sea el punto de partida para que las mismas universidades pongan, de acuerdo a su contexto y presupuesto, las metas a conseguir en los siguientes años, las cuales deberían ser asequibles pero no muy bajas para realmente motivar a realizar esfuerzos por conseguir una universidad socialmente responsable.

Finalmente, se muestra la urgencia de las facultades de negocios por incorporar iniciativas de RSU para formar a sus egresados, futuros líderes y tomadores de decisiones en empresas, para mejorar hacia una gestión sustentable de empresas y con esto disminuir las probabilidades de futuros escándalos financieros, malas prácticas, colusión, entre otros. Además, que si mejoran en aspectos relacionados a RSU estarán también ayudando a mejorar la calidad institucional por los criterios de acreditación en Chile.

Es importante mencionar que es un proceso gradual y se debe empezar por cambios organizacionales sencillos, donde lo principal es que la gestión interna de la universidad sea reflejo de democracia, equidad, transparencia y que se pueda realizar bajo un modelo constante y sostenible en el tiempo. 


\section{REFERENCIAS BIBLIOGRÁFICAS}

AUSJAL, (2009). "Políticas y sistema de autoevaluación y gestión de la responsabilidad social universitaria en AUSJAL". AUSJAL, Argentina.

Kaivola, T., \& Rohweder, L, (2007). “Towards Sustainable Development in Higher EducationReflections". Publications of the Ministry of Education. Helsinki: Helsinki University Press.

ONU, (2015). “Objetivos del desarrollo sostenible". Extraído el 13 de Noviembre 2015 desde: http:/ / www.un.org/sustainabledevelopment/ es/objetivos-de-desarrollo-sostenible /

UNESCO, (1998). Declaración Mundial Sobre la Educación Superior en el siglo XXI: Visión y Acción.

Universidad Construye-País, (2002). "Hacia las sociedades del conocimiento, informe mundial. Observando la Responsabilidad Social Universi- taria", Documento de Trabajo, Santiago de Chile.

Universidad Construye-País, (2006). "Responsabilidad Social Universitaria; Una manera de ser Universidad, Teoría y práctica de la experiencia Chilena", Documento de Trabajo, Santiago de Chile.

Vallaeys, F., (2006). "Responsabilidad social Universitaria: Hacia un concepto maduro para su gestión institucional". Pontificia Universidad Católica de Perú.

Vallaeys, F., (2009). "Responsabilidad Social Universitaria, Manual de Primeros Pasos" BID / McGrawHill.

Vallaeys, F., (2013). La Responsabilidad Social Universitaria: ¿Cómo entenderla para quererla y practicarla? Revista Servicio Comunitario.

\section{ANEXO}

1. Tabla I: Universidades participantes en UCP

\begin{tabular}{|c|c|}
\hline \multicolumn{2}{|c|}{ Universidades participantes Proyecto Universidad Construye País } \\
\hline Universidad de Chile & Universidad Técnica Federico Santa María \\
\hline Pontificia Universidad Católica de Chile & Universidad Playa Ancha \\
\hline Universidad Alberto Hurtado & Pontificia Universidad Católica de Valparaíso \\
\hline Universidad de Santiago de Chile & Universidad de Valparaíso \\
\hline Universidad de La Frontera & Universidad de Concepción \\
\hline Universidad Católica de Temuco & Universidad del Bío - Bío \\
\hline Universidad Austral de Chile & \\
\hline
\end{tabular}

Fuente: Creación propia. 